\title{
3D white graphene foam scavengers: vesicant-assisted foaming boosts the gram-level yield and forms hierarchical pores for superstrong pollutant removal applications
}

\begin{abstract}
Huijie Zhao ${ }^{1,2}$, Xiufeng Song ${ }^{1}$ and Haibo Zeng ${ }^{1,2}$
Three-dimensional (3D) nanostructures assembled with one- or few-layered ultrathin two-dimensional (2D) crystals have triggered great interest in energy and environmental applications. Here, we introduce a gas-foaming process in an hexagonal boron nitride (h-BN) ceramic material to fabricate 3D white graphene (WG) foams without using any catalysts or templates for superstrong pollutant removal applications. Importantly, the introduction of vesicants guaranteed the reproducibility and yield $\left(>500 \mathrm{~cm}^{3}\right)$. Interestingly, these 3D WG foams possessed a vesicular structure with hierarchical pores ranging from $\mathrm{nm}$ to $\mu \mathrm{m}$ scales and with ultrathin walls consisting of mono- or few-layered BN membranes with planar sizes as large as $100 \mu \mathrm{m}$. Consequently, such microstructure merits of hierarchical pores and ultrathin walls endowed them not only very low density $\left(2.1 \mathrm{mg} \mathrm{cm}^{-3}\right)$ but also superstrong adsorption ability, illustrated by capacitances up to 190 times its own weight toward a wide range of environment contaminations, including various oils and dyes. Thus, the 3D h-BN WG foams prepared by vesicant-assisted foaming should have great potential as outstanding environmental scavengers.
\end{abstract}

NPG Asia Materials (2015) 7, e168; doi:10.1038/am.2015.8; published online 27 March 2015

\section{INTRODUCTION}

Two-dimensional (2D) crystals, such as graphene ${ }^{1}$ and white graphene (WG, mono- or few-layered hexagonal boron nitride (h-BN)), ${ }^{2,3}$ have triggered great interest because of their extraordinary intrinsic properties and wide range of applications in electronics, optoelectronics, energy storage and the environment. ${ }^{4}$ However, for some specific applications, such as the adsorption of various contaminants and as electrodes in electrochemical cells, their pristine flat $2 \mathrm{D}$ structures have been recognized to not fully match the practical requirements. ${ }^{5-8}$ In contrast, three-dimensional (3D) architectures using 2D crystals as building blocks can simultaneously provide the virtues of 2D and 3D structures, such as ultrathin sheets and large specific surface areas from 2D sheets ${ }^{6}$ and hierarchical pores and ultralight densities from 3D configurations. ${ }^{7}$ Recently, such novel 2D-3D structure features have been proven to exhibit new and outstanding performances. For instance, graphenecarbon nanotube 3D structures had densities as low as $0.16 \mathrm{mg} \mathrm{cm}^{-3}$, even lighter than air $\left(1.29 \mathrm{mg} \mathrm{cm}^{-3}\right) ;{ }^{9} 3 \mathrm{D}$ graphene and $\mathrm{BN}$ networks exhibited excellent mechanical properties; ${ }^{10,11}$ 3D BNC hybrid networks showed tunable electronic and thermal properties. ${ }^{12}$

However, the high-yield fabrication of such $3 \mathrm{D}$ architectures of $2 \mathrm{D}$ crystals, especially without using any templates or catalysts, remains a great challenge. Currently, there are two methods for fabricating $3 \mathrm{D}$
WG foams. The first method involves assembling chemically prepared $2 \mathrm{D}$ sheets into $3 \mathrm{D}$ structures. ${ }^{5-9}$ Clearly, the yield is limited by the primal $2 \mathrm{D}$ crystals and the assembly process. More importantly, the artificial and poor connections among the $2 \mathrm{D}$ crystals usually degrade the electrical and thermal transport inside such $3 \mathrm{D}$ structures. ${ }^{13-16}$ To achieve naturally integrated 3D networks, a second method was recently developed. ${ }^{17}$ Chen et al. ${ }^{10}$ reported the chemical vapor deposition growth of porous graphene 3D structures with nickel foam as a catalyst and $3 \mathrm{D}$ template. This chemical vapor deposition approach can provide high mechanical and electrical properties and hence has attracted much interest in energy devices. ${ }^{11,12,17}$ However, the yield and cost is limited by the use of Ni foams. According to the current state in this field, the realization of high-yield and natural connections will greatly advance 3D WG foams. However, a facile and large-yield method without the use of any catalysts or templates is still absent. Importantly, the introduction of vesicants guarantees the reproducibility and yield $\left(>500 \mathrm{~cm}^{3}\right)$.

Here, for the first time, we report a vesicant-assisted foaming method for the high-yield fabrication of 3D WG foams possessing superhigh adsorption capacities without the use of any templates. The vesicants were intricately matched to the decomposing thermodynamics of the raw materials to boost the foaming effect, resulting in

${ }^{1}$ Institute of Optoelectronics and Nanomaterials (ION) and Herbert Gleiter Institute of Nanoscience (HGI), College of Materials Science and Engineering, Nanjing University of Science and Technology, Nanjing, China and ${ }^{2}$ College of Materials Science and Technology, Nanjing University of Aeronautics and Astronautics, Nanjing, China

Correspondence: Professor H Zeng, School of Materials Science and Engineering, Nanjing University of Science and Technology, 200 Xiaolingwei Street, Nanjing, Jiangsu 210094, China.

E-mail: zeng.haibo@njust.edu.cn

Received 8 October 2014; revised 12 December 2014; accepted 23 December 2014 
3D architectures consisting of few-layered $\mathrm{BN}$ walls and hierarchical pores with sizes ranging from several $\mathrm{nm}$ to several hundred $\mu \mathrm{m}$. Such novel structural features endowed the 3D WG foam with the strong ability to absorb various dyes and organic pollutants. Typically, 3D WG foam with a density of $2.1 \mathrm{mg} \mathrm{cm}^{-3}$, which was 1000 times less than bulk h-BN, could absorb 190 times their weight in pump oil, the highest capacity achieved when compared with reported $\mathrm{BN}$ materials and commercial activated carbon sorbents. It should be noted that the method adopted simple heating mixtures of raw materials and vesicants; therefore, it is very facile and should have a limitless yield. Hence, this method could be easily scaled up for future industrial manufacture.

\section{EXPERIMENTAL PROCEDURE}

\section{Preparation of the 3D WG foams}

The 3D WG foams were synthesized in a horizontal electric furnace with a fused alumina tube $100 \mathrm{~cm}$ in length and $6 \mathrm{~cm}$ in diameter. Initially, $200 \mathrm{mg}$ ammonia borane $(\mathrm{AB})$ and $40 \mathrm{mg}$ thiourea (or aminothiourea) were mechanically mixed, and the mixed powers as precursors were placed into an alumina crucible. The alumina crucible with the precursors was loaded into an alumina tube. The alumina tube was evacuated to $10 \mathrm{~Pa}$, and nitrogen gas was subsequently introduced into the tube at a rate of 100 SCCM. The precursors were pretreated at $80^{\circ} \mathrm{C}$ for $1 \mathrm{~h}$. Then, the temperature was increased to $1200^{\circ} \mathrm{C}$ at a rate of $15^{\circ} \mathrm{C} \mathrm{min}^{-1}$ and was maintained at that temperature for $\sim 2 \mathrm{~h}$ to synthesize 3D WG foams. After the tube was cooled to room temperature, the final foamed products with white color were obtained.

\section{Characterizations of the 3D WG foams}

Powder X-ray diffraction patterns of the obtained materials were recorded on Bruker D8 diffractometer (Karlsruhe, Germany) with $\mathrm{Cu} \mathrm{K}_{\alpha}$ radiation $(\lambda=1.5406 \AA)$ at $40 \mathrm{kV}$ and $20 \mathrm{~mA}$. The morphologies of the prepared materials were examined using field-emission scanning electron microscopy (JSM-6701F, JEOL, Kawagoe, Japan) and atomic force microscopy (Multimode 8, Bruker). Transmission electron microscopy images and electron energy loss spectroscopy spectra were recorded using a FEI Tecnai G2 20 at $200 \mathrm{KV}$ (Hillsboro, OR, USA). Fourier transform infrared spectra were obtained on a Bruker Vector 22 FT-IR spectrometer in the range of $400-4000 \mathrm{~cm}^{-1}$ using a standard $\mathrm{KBr}$ disk. Raman scattering spectra were obtained using a Horiba Jobin Yvon LABRAMHR800 laser micro-Raman spectrometer (Tokyo, Japan) with a $532 \mathrm{~nm}$ laser. Ultraviolet-visible (UV-vis) absorption spectra were recorded using a UV-3600 UV-vis spectrophotometer (Shimadzu, Kyoto, Japan). Thermogravimetric and differential thermal analysis were carried out using a thermogravimetric analyzer (Perki Elimer Pyris 1, Waltham, MA, USA) from room temperature to $800^{\circ} \mathrm{C}$ at a heating rate of $10^{\circ} \mathrm{C} \mathrm{min}^{-1}$ under a $\mathrm{N}_{2}$ atmosphere. The specific surface areas and pore structures were obtained from the adsorption isotherms of nitrogen at $77 \mathrm{~K}$ in a Quantachrome Autosorb-6B apparatus using the Brunauer-Emmett-Teller and Barratt-Joyner-Halenda methods, respectively.

\section{Adsorption performance measurements}

Adsorption experiments were carried out to investigate the adsorption behaviors of the obtained 3D WG foams. In a typical procedure, $100 \mathrm{mg} \mathrm{BN}$ was added to a $250 \mathrm{ml}$ methyl blue (MB) aqueous solution $\left(120 \mathrm{mgl}^{-1}\right)$ while stirring. The MB concentration was examined at different intervals by UV-vis absorption (Shimadzu UV-3600).

The Langmuir adsorption model was used to characterize the adsorption capacity of a sorbent for pollutants. The Langmuir isotherm is represented as follows:

$$
Q_{\mathrm{e}}=Q_{\mathrm{m}} K C_{\mathrm{e}} /\left(1+K C_{\mathrm{e}}\right)
$$

where $Q_{e}\left(\mathrm{mgg}^{-1}\right)$ is the adsorbed amount of dyes at the equilibrium concentration $\left(\mathrm{mg} \mathrm{g}^{-1}\right), C_{\mathrm{e}}\left(\mathrm{mgl}^{-1}\right)$ is the equilibrium solute concentration, $Q_{\mathrm{m}}$ is the maximum adsorption capacity corresponding to complete monolayer coverage and $K$ is the equilibrium constant $\left(\mathrm{mg}^{-1}\right)$.

To measure the adsorption capacities for oils and organic solvents, the 3D WG foams were weighed and then placed into the oils and organic solvents.
After adsorption was complete, the $\mathrm{BN}$ was removed from the oil and weighed again. The adsorption capacity $(Q)$ was calculated using the following equation:

$$
Q=\left(M-M_{0}\right) / M_{0}
$$

where $M_{0}$ and $M$ are the weights of the WG foams before and after adsorption, respectively. The weight measurements of the $\mathrm{BN}$ with absorbed oil or organic solvent were quickly performed to avoid evaporation of the oils or organic solvents.

\section{RESULTS AND DISCUSSION}

Foaming effects can be frequently observed in a variety of daily events (typically the formation of soap and beer foam bubbles) and have been historically applied to fabricate polymer and metal foams. Normally, gas bubbles formed by vesicants in a metallic melt tend to quickly rise to the surface because of the high buoyancy forces in the high-density liquid, and this rise can be hampered by the surface tension force. ${ }^{18,19}$ The imbalance between the buoyancy and the surface tension force determines the size of the gas bubbles in the metallic melts and the pore size in the final solid foams. ${ }^{19}$

According to basic principle above, three factors are prerequisite to produce foams: an intermediate liquid state, energetic gas-releasing vesicants and thermodynamic matching between the vesicant and the raw materials. These chemical foaming factors have been well documented for polymer and metal foams. Typically, for polymer foams, the in situ decomposition reactions of isocyanate, hydrazine and sodium bicarbonate are well matched to the solidliquid-solid phase changes of various polymers at relatively low temperature. ${ }^{20}$ In contrast, for metal foams, titanium hydride and zirconium hydride decompose to form gases at elevated temperatures. $^{20,21}$

However, for ceramic materials, the corresponding foaming process is far from developed. The main reason is because melting temperatures of ceramics $\left(2050{ }^{\circ} \mathrm{C}\right.$ for $\mathrm{Al}_{2} \mathrm{O}_{3}, 3300^{\circ} \mathrm{C}$ for h-BN) are much higher than those of typical polymers $\left(200-400^{\circ} \mathrm{C}\right)$ and metals $\left(660^{\circ} \mathrm{C}\right.$ for $\mathrm{Al}, 1455^{\circ} \mathrm{C}$ for $\mathrm{Ni}$ ), causing great difficulty in identifying the appropriate vesicants for the melted matrix materials. In contrast, ceramic foams formed by impregnating polymer foams with a ceramic slurry followed by firing have been promising for a variety of applications, such as acoustic insulation, as substrates for catalysts requiring large internal surface areas and for adsorbing environmental pollutants. 22,23

According to above foaming factors and difficulties for ceramics, the foaming process and formation of 3D BN WG foams is illustrated in Figure 1a. Several issues were the key points for the designed method here. First, $\mathrm{AB}$ was used as the raw material, replacing the direct usage of matrix materials in traditional polymer and metal foaming processes. ${ }^{24-26}$ This precursor selection avoided the unrealizable high foaming temperature of $>3300^{\circ} \mathrm{C}$. AB can be transformed into a B$\mathrm{N}-\mathrm{H}$ polymeric liquid derivate at temperatures as low as $100-200^{\circ} \mathrm{C} .^{27}$ This conversion has been previously reported and was further evidenced here by thermogravimetry, as shown in Figure $1 \mathrm{~b}$. The mass of $\mathrm{AB}$ decreased $\sim 55 \%$ below $200{ }^{\circ} \mathrm{C}$ because of the release of $\mathrm{H}_{2}$, leaving behind the polymer-like aminoborane and polyiminoborane. ${ }^{27}$ Second, thiourea and aminothiourea were selected as typical vesicants. Figure $1 \mathrm{~b}$ demonstrates that the gases $\left(\mathrm{CS}_{2}, \mathrm{NH}_{3}\right.$ and NCNS) released at $200-300{ }^{\circ} \mathrm{C}$ reached $65 \%$ and $80 \%$ weight of the primal thiourea and aminothiourea, respectively. Such approximate matching temperature was crucial for the formation of metastable gas bubbles in the intermediate state of the $\mathrm{AB}$ precursor. Furthermore, the sufficient gas release from the vesicants compensated 

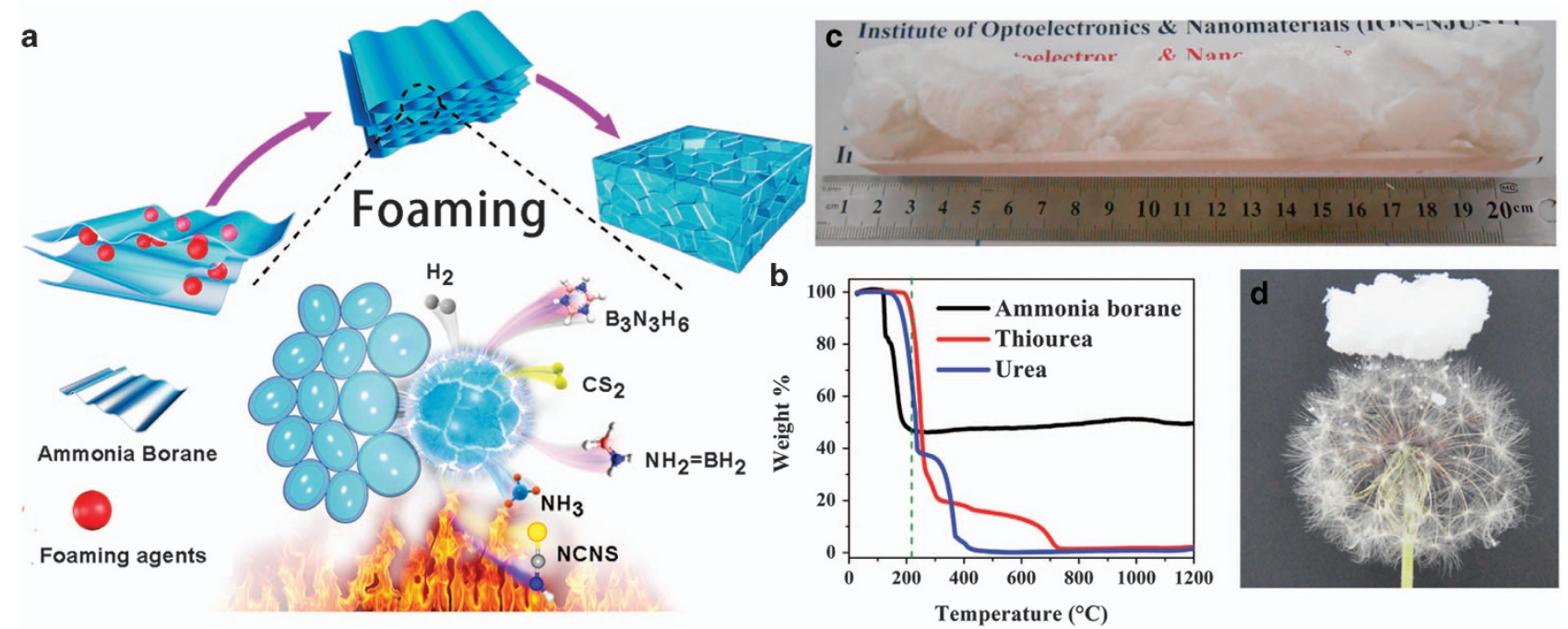

Figure 1 (a) Schematic illustration of vesicant-assisted foaming of three-dimensional (3D) white graphene (WG) foams. The gas released from the vesicants leads to the bubbles in the intermediate polymeric derivate and then the hexagonal boron nitride (h-BN) foams. (b) Thermogravimetric (TG) curves of BN precursors (ammonia borane) and vesicants (thiourea and aminothiourea). (c) Photograph of an as-prepared 3D WG foam. (d) A piece of a WG foam standing on a dandelion.

for the insufficient decomposition of the $\mathrm{AB}$ precursor and hence guaranteed the large void volume in the intermediate state. As the temperature was elevated further, the void-impregnated intermediate products tended to crystallize in situ. Then, they were gradually transformed into 3D WG foams with a mass of pores and ultrathin h-BN walls.

Clearly, when compared with previous methods for fabricating 3D architectures consisting of 2D crystals, either the assembly of 2D sheets or chemical vapor deposition growth in $3 \mathrm{D} \mathrm{Ni}$ foams, the foaming approach achieved by heating the mixtures of $\mathrm{AB}$ and thiourea exhibited remarkably superior yield. The starting materials were all powders and there was no usage of metal foams, indicating that the traditional limitations from $2 \mathrm{D}$ sheet precursors and 3D templates were overcome in this vesicant-assisted foaming method. Therefore, very high yield could be easily achieved, and the only limitation was the volume of the used container, as shown in Supplementary Figure S1. Figure 1c exhibits a typical 3D WG foam with a length of $20 \mathrm{~cm}$. The volume expanded several hundred times when compared with the raw materials, and hence the density decreased to as low as 2.1 $\mathrm{mg} \mathrm{cm}{ }^{-3}$, near that of air $\left(1.275 \mathrm{mg} \mathrm{cm}^{-3}\right)$. The formed 3D WG foams were typical ultralight materials (defined as a density of $<10$ $\mathrm{mg} \mathrm{cm}{ }^{-3}$ ), and could even freely stand on a dandelion flower, as shown in Figure 1d.

For solid foams, the details of the inside of the pores are the most important structural features. Figure 2 reveals Plateau's laws of typical foam materials and hierarchical pores in the $3 \mathrm{D}$ WG foams. ${ }^{28,29}$ Figure $2 \mathrm{a}$ presents a typical cross-section that illustrates the structure inside the 3D WG foam. Clearly, the foams consisted of $20-100 \mu \mathrm{m}$ pores and interconnected walls. Most of the pores had an irregular hexagonal structure. Three edges always met in a so-called Plateau border with an angle of $\sim 120^{\circ} .28,30$ The intact top surfaces in Figure $2 \mathrm{~b}$ exhibited another feature of Plateau's laws: the walls were complete and smooth surfaces with a mean curvature of a constant on any point. Furthermore, Figure 2c demonstrates that the detached sheets had very smooth surfaces and a planar size of several tens of $\mu \mathrm{m}$, coincident with Figure 1b. However, the thickness was very low, indicating ultrathin walls that originated from the sufficient foaming effect. The foams obeyed Plateau's laws because of the requirement for minimal total surface area that has been mathematically proven by Jean Taylor using the geometric measure theory. ${ }^{31}$ Other configurations are unstable and tend to quickly rearrange the bubbles in the intermediate state during the foaming process into this type of structure. The matching to Plateau's laws strongly evidenced that the designed foaming process as shown in Figure 1a indeed occurred and resulted in these 3D WG foams. ${ }^{28,29}$

Interestingly, in addition to $\mu \mathrm{m}$-scale pores, nm-scale pores were also very common in these ultrathin walls when observed by transmission electron microscopy, as shown in Figure 2d. These pores had a bimodal size distribution of $\sim 60$ and $5 \mathrm{~nm}$. Actually, the emergence of these small pores was reasonable and even solved an inconsistency between the foaming principle in Figure 1a and the Plateau structure in Figure 2a and b. According to Figure 1b, the gas released from vesicants starts at the polymer-like intermediate state of the $\mathrm{BN}$ precursors and slightly persists until the crystallization stage above $200^{\circ} \mathrm{C}$. Therefore, the persistent gas releasing should result in open-cell foams; however, only closed-cell foams were revealed in Figure $1 \mathrm{a}$ and $\mathrm{b}$. Figure 1d proves that a mass of small pores on the ultrathin walls could provide the releasing channels for the superfluous gases from residual effective vesicants. The formation of these small pores was because of the persistent gas release from the residual vesicant particles that could enlarge and adsorb onto the preformed ultrathin walls.

The nm-scale pores were further verified by Barratt-JoynerHalenda measurements from the nitrogen sorption characterization, as shown in Figure 2e. Clearly, there were some pores with sizes in the range of 5-150 nm with a peak at $\sim 10 \mathrm{~nm}$ and long tail at $50-120 \mathrm{~nm}$. These pore features were accordant with the transmission electron microscopy result shown in Figure $2 \mathrm{~d}$. The $\mu \mathrm{m}$-scale pores were not revealed by Barratt-Joyner-Halenda because they were not sensitive to the surface adsorption-desorption process. Taken together, the above analyses demonstrated that the 3D WG foams had hierarchical pores, as shown in Figure 2f, with three distribution peaks at $50 \mu \mathrm{m}, 60 \mathrm{~nm}$ and $10 \mathrm{~nm}$ (labeled as I, II and III). This is the first report of such 

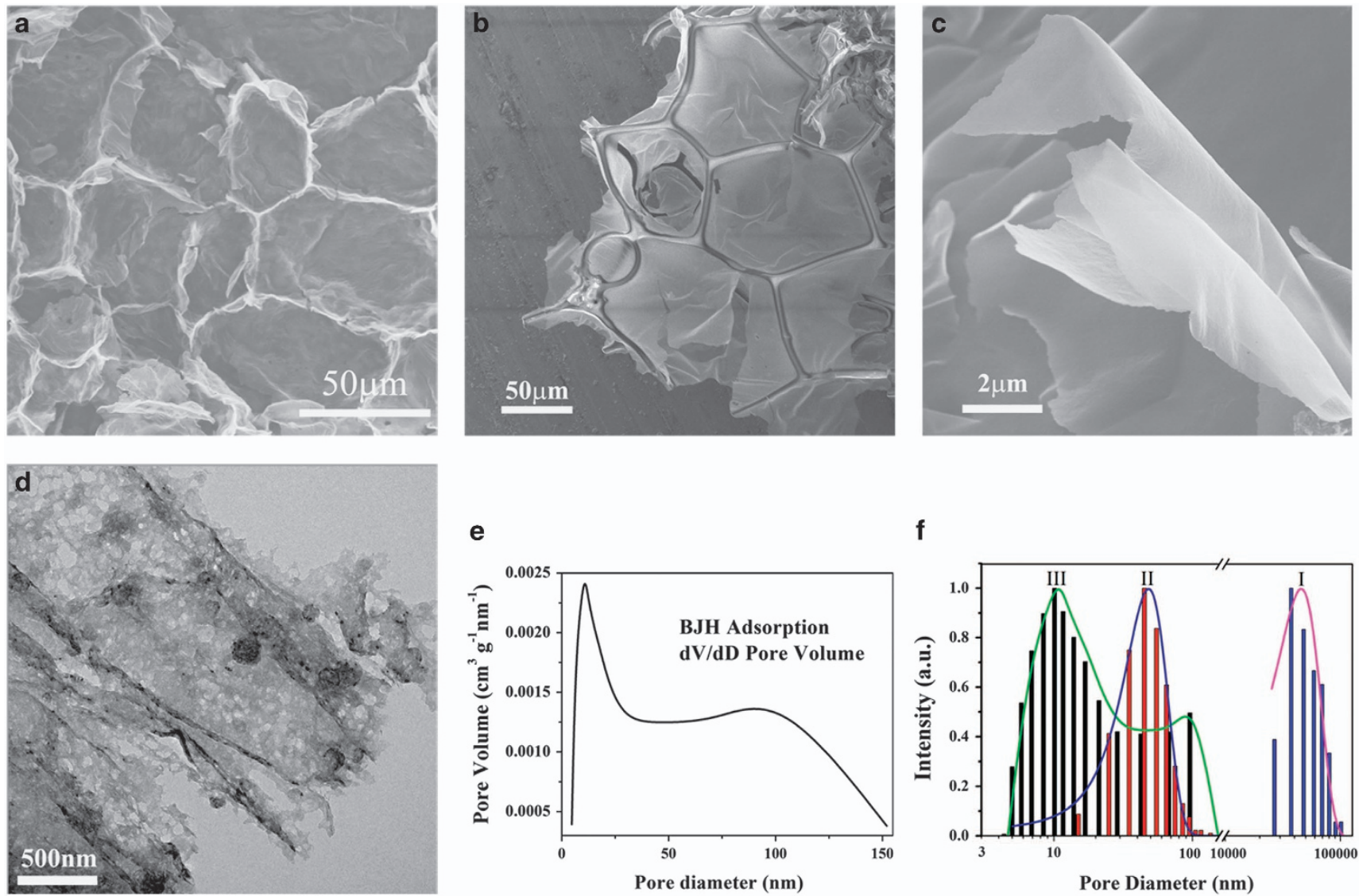

e

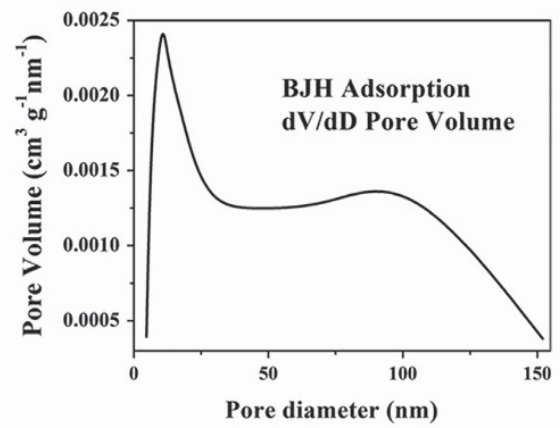

$\mathbf{f}$

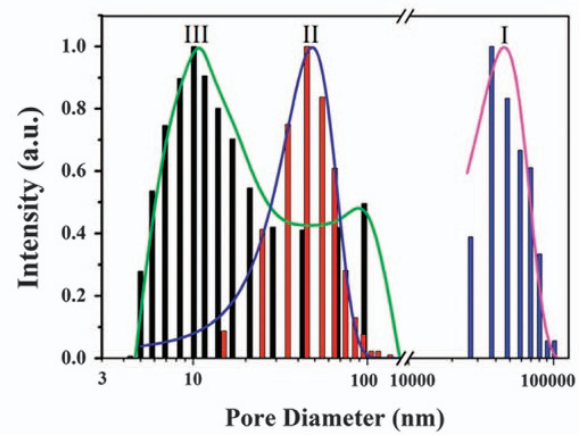

Figure 2 Hierarchical pores inside three-dimensional (3D) white graphene (WG) foams. (a) Cross-section and (b) top-view scanning electron microscopy (SEM) images of freestanding 3D WG foams, showing 20-100 $\mu \mathrm{m}$ pores. (c) SEM image of boron nitride (BN) sheets separated from WG foams, showing ultrathin thicknesses and similar transverse sizes to the pore walls. (d) Transmission electron microscopy (TEM) image of BN sheets with 30-65 nm pores. (e) The pore size distribution of WG foams according to the Barratt-Joyner-Halenda (BJH) adsorption. (f) The statistical size distribution of the hierarchical pores in WG foams, showing three peaks at $6 \mathrm{~nm}, 48 \mathrm{~nm}$ and $45 \mu \mathrm{m}$. The data of curves I and II from the statistical analysis of the SEM and TEM images, respectively. Curve III results from (e).

hierarchically porous structure in $3 \mathrm{D}$ structured $\mathrm{BN}$ and graphene. This structure was because of the vesicant-assisted foam formation and the slightly different decomposition thermodynamics between the vesicants and precursors, as discussed above. Importantly, these 3D hierarchical pores could greatly benefit some applications, such as pollutant removal, as demonstrated below.

Following the hierarchical pore features, the ultrathin h-BN walls of the 3D WG foams were characterized in Figure 3. Most separated walls had layer numbers $<10$, as demonstrated by the typical one-layer sheet in Figure $3 \mathrm{a}$ and the 2-3-layer sheet in Figure $3 \mathrm{~b}$. The edges in Figure $3 \mathrm{~b}$ showed an interlayer distance of $0.33 \mathrm{~nm}$, consistent with the (002) crystal planes of h-BN. Figure $3 c$ presents the honeycomb lattice with a $0.25-\mathrm{nm}$ distance between two atoms and the corresponding fast Fourier transform pattern. These transmission electron microscopy (Supplementary Figure S2) and X-ray diffraction (Supplementary Figure S3) results were in agreement with the lattice structure of layer-structured hexagonal BN. Furthermore, the atomic force microscopy image and height profile in Figure 3d exhibited several sheets with thicknesses as low as $1 \mathrm{~nm}$, corresponding to one or two atomic layers (Figure 3d), indicating the ultrathin characteristic of the walls in the 3D WG foams. The chemical composition and stoichiometry of $\mathrm{BN}$ were verified using electron energy loss spectroscopy, as shown in Figure 3e. The electron energy loss spectroscopy peaks at $180-220 \mathrm{eV}$ and $400-430 \mathrm{eV}$ corresponded to the characteristic K-shell ionization edges of $\mathrm{B}$ and $\mathrm{N}$ atoms with a B-to- $\mathrm{N}$ ratio of 1:1. Their split fine superstructures confirmed that the obtained products were typical $\mathrm{sp}^{2}$-hybrizied h-BN. ${ }^{32}$ The B-N bonding could also be well confirmed by the X-ray photoelectron (Supplementary Figure S4), Raman (Supplementary Figure S5) and Fourier transform infrared (Supplementary Figure S6) spectra. The optical band-gap obtained from the UV-vis absorption spectrum (Supplementary Figure S7) was $\sim 5.98 \mathrm{eV}$.

Three-dimensional ceramic foams have been considered to be excellent sorbents for various environment pollutants, such as dyes and organics. ${ }^{22}$ However, for nanomaterials, their low yield is usually a fatal drawback for such applications. Furthermore, pores inside the sorbents are needed to enhance their adsorption capacity. Here, the vesicant-assisted foaming strategy resulted in high-yield formation and hierarchical pores inside 3D WG foams. These two significant aspects of $3 \mathrm{D}$ WG foams led them to be proposed for high-performance pollutant removal applications.

First, MB, a typical cationic industrial dye, was used as a model material to confirm the adsorption capacity of the 3D WG foams. Figure $4 \mathrm{a}$ shows the variation of the UV-vis absorption spectra after dipping the 3D WG foams into a MB solution. Such spectra changes were transformed into the concentration decrease of the residual $\mathrm{MB}$ 

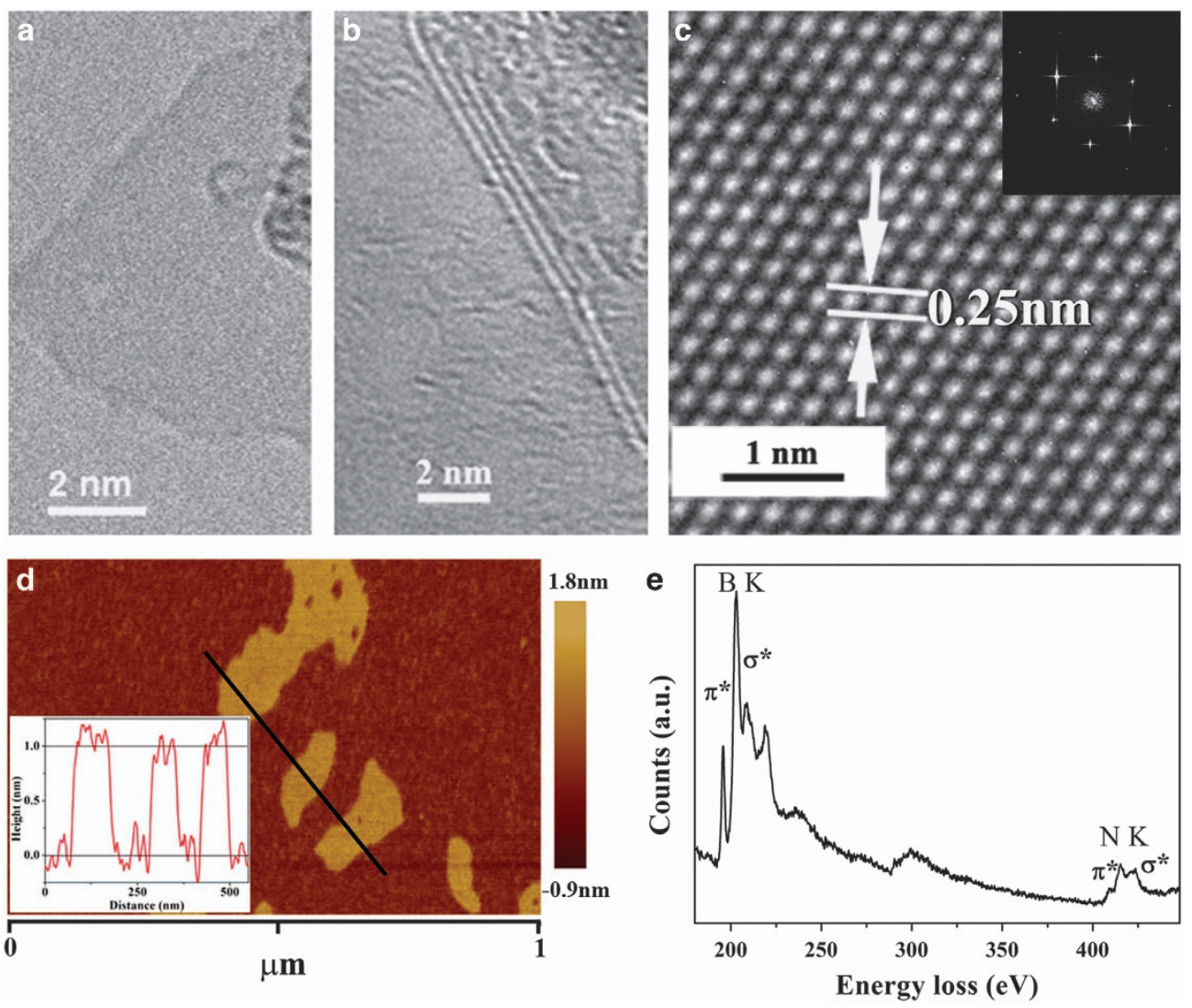

Figure 3 Ultrathin walls of three-dimensional (3D) white graphene (WG) foams. High-resolution transmission electron microscopy (HRTEM) images of (a) monolayer edges, (b) few-layer edges and (c) surface lattice fringes and fast Fourier transform (FFT) patterns of ultrathin boron nitride (BN) sheets. (d) Atomic force microscopy (AFM) image and height profile of BN sheets. (e) Electron energy loss spectroscopy (EELS) spectrum of BN sheets.

in the solution, as shown in Figure $4 \mathrm{~b}$. Clearly, the MB adsorption from the 3D WG foams was not only very fast but also very thoroughgoing. Most of the MB molecules ( 94\%) were adsorbed within $20 \mathrm{~min}$, and all of them were exhausted after $1 \mathrm{~h}$. Actually, this outstanding adsorption ability of 3D WG foams was visual, as shown in the inset of Figure $4 \mathrm{~b}$. The blue color, corresponding to $\mathrm{MB}$ molecules, rapidly disappeared when the $3 \mathrm{D}$ WG foams were immersed into the solution. At the same time, the color of $3 \mathrm{D}$ WG foams changed from snow white to blue, as shown in the inset of Figure 4c. The quantized adsorption capacity was shown in Figure 4c according to the weights of adsorbed $\mathrm{MB}$ and the tested foams. The maximum MB capacity of the 3D WG foams was near $500 \mathrm{mg} \mathrm{g}^{-1}$ (accurately $497 \mathrm{mg} \mathrm{g}^{-1}$ ). Interestingly, after heating at $400{ }^{\circ} \mathrm{C}$ for $2 \mathrm{~h}$ in air, all of adsorbed MB molecules were released, and these 3D WG foams recovered their white color and high adsorption ability (slightly reduced to $461 \mathrm{mgg}^{-1}$, Supplementary Figure S8), indicating their potential as recyclable environment cleaners. Such strong adsorption ability was validated again when the $\mathrm{MB}$ was changed to another typical anionic industrial dye, methyl orange (Supplementary Figure S9). These 3D WG foams exhibited the highest adsorption capacity to dyes that was much higher than previously reported values, as shown in Figure 4d, when compared with similar sorbents, including BN ultrathin hollow spheres $\left(116.5 \mathrm{mgg}^{-1}\right),{ }^{33} \mathrm{BN}$ ultrathin fibrous nanonets $\left(219.6 \mathrm{mg} \mathrm{g}^{-1}\right),{ }^{34} \mathrm{BN}$ nanocarpets $\left(272.4 \mathrm{mg} \mathrm{g}^{-1}\right)^{35}$ and $\mathrm{BN}$ porous nanosheets $\left(313 \mathrm{mg} \mathrm{g}^{-1}\right) .{ }^{36}$

In addition to typical dyes, various organics and oils are more common daily and industrial pollutants. Surprisingly, these 3D WG foams exhibited much higher adsorption capacities to these organics and oils compared with many competitors, as shown in Figure 5. The rapid and effective adsorption behaviors were directly visualized, as shown in Figure 5a and Supplementary Movie S1. After dipping the 3D WG foams into an aqueous solution of chloroform, which was intentionally stained with Sudan III to mark the foams red in color, these red pollutants absorbed rapidly and completely within $2 \mathrm{~min}$. To confirm the universality of the adsorption ability of the 3D WG foams, a series of organics and oils, such as toluene, pump oil, ethanol, chloroform and ethylene glycol, were selected for adsorption measurements. Figure $5 \mathrm{~b}$ clearly presents the adsorption capacities of the $3 \mathrm{D}$ WG foams to different pollutants in the range of 70-190 times its own weight, demonstrating an outstanding adsorption ability to all of the mentioned organics.

For pump oil, a common pollutant, the typical adsorption capacity of 3D WG foams was as high as $115 \mathrm{~g} \mathrm{~g}^{-1}$, as shown in Figure 5b, and this means that only $1 \mathrm{~kg}$ of $3 \mathrm{D}$ WG foams is needed to purify 1 ton of water containing $10 \%$ oil pollutant. To more quantitatively evaluate the adsorption capacity of 3D WG foams, several recently reported excellent sorbents and commercial activated carbon powders were adopted for comparison, as shown in Figure 5c. Taking the adsorption of pump oil as an example, the adsorption capacity $\left(115 \mathrm{gg}^{-1}\right)$ of the 3D WG foams was higher than all of these competitors, including BN porous nanosheets $\left(27 \mathrm{gg}^{-1}\right),{ }^{36}$ graphene aerogels $\left(30 \mathrm{gg}^{-1}\right),{ }^{14}$ graphene sponges $\left(68.5 \mathrm{gg}^{-1}\right)^{8}$ and graphene oxide-polyurethane sponges $\left(100 \mathrm{~g} \mathrm{~g}^{-1}\right)^{16}$ (see Supplementary Table S1). Compared with commercial $\mathrm{BN}$ and active carbon powders, the adsorption capacity 
a

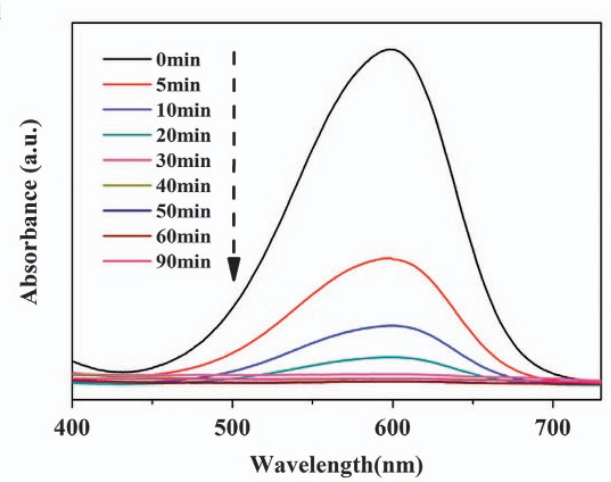

c

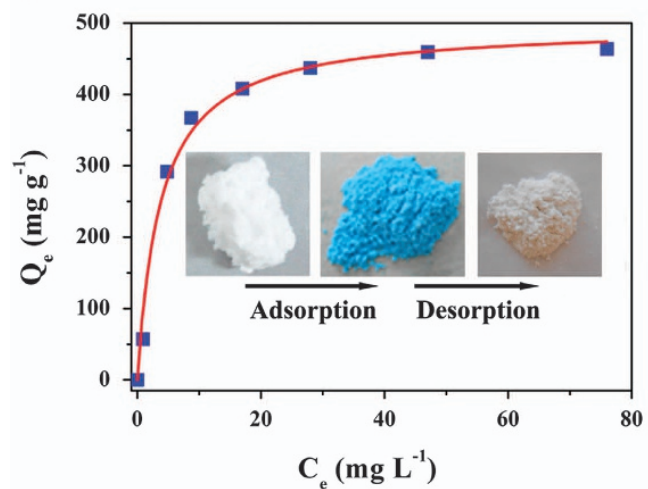

b

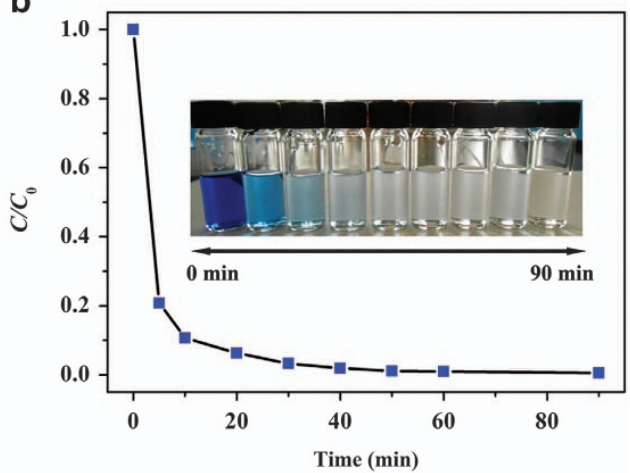

d

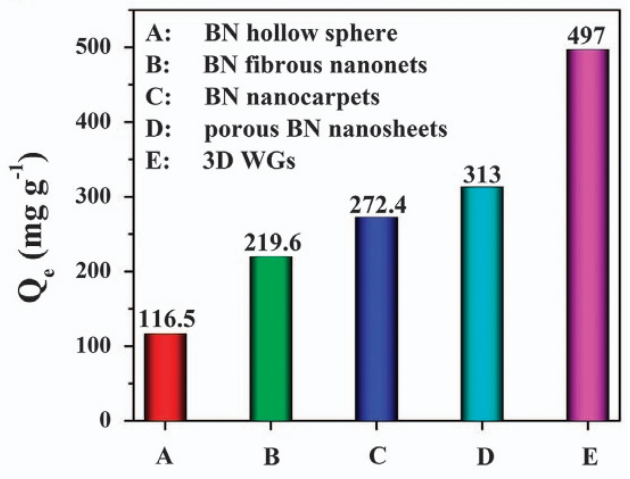

Figure 4 Strong adsorption of dyes on three-dimensional (3D) white graphene (WG) foams. (a) Evolution of the absorption spectra of the 3D WG-immersed methyl blue (MB) solution with time. (b) The corresponding adsorption rate and visual variation of the MB solution with time. (c) MB adsorption isotherm and adsorption-desorption recycling of 3D WG foams. (d) Comparison of the MB adsorption capacities of 3D WG foams with various reported boron nitride (BN) nanostructures.

of the 3D WG foams was $>20$ times larger than these typical sorbents.

Recyclability, the important characteristic of sorbents, was tested for these 3D WG foams, as shown in Figure $5 \mathrm{~d}$ and e. After 7 test cycles and heat treatments at $100^{\circ} \mathrm{C}$, the adsorption capacities of the 3D WG foams still remained $>90 \%$ toward both chloroform and ethanol. For pump oil, the removal efficiency was still maintained at $\sim 85 \%$ even after five cycles through direct combustion in air (Supplementary Figure S10). Clearly, these results demonstrated that the obtained 3D WG foams could be considered highly effective and recyclable sorbents for the removal of pollutants in environment purification.

The superstrong competitive power of 3D WG foams over other sorbents arose from their two crucial features, large-yield formation and hierarchical pores, that both profited from the vesicant-assisted foaming. Just because of the special structure with hierarchical pores, the 3D WG presented outstanding pollutant removal properties. First, the superthin BN layers with many ripples and fluctuations were excellent sorbents for organic solvents. Second, there were many holes in the surfaces of the $\mathrm{BN}$ walls, and the edges of the holes resulted in high-density defects that could provide numerous active sites for the adsorption of pollutant molecules onto the BN surface. Third, the small holes in the surface of the $\mathrm{BN}$ wall provided a transport channel for pollutants in BN sheets, similar to the opened foam structure. Compared with the infiltration mechanism from the $\mathrm{BN}$ sheets, these channels allow pollutants to easily enter the $3 \mathrm{D}$ WG foams and improve their adsorption efficiency. Fourth, after the organic solvents entered the bubbles of the $\mathrm{BN}$ foams, the bubble could be considered a vessel to store the organic solvents. The storage of organic solvents in bubbles was much greater than that adsorbed on the surfaces of the bubble walls, the crucial factor for the outstanding pollutant removal properties of these 3D WG foams compared with other BN nanosheets. ${ }^{33,35-38}$ This pivotal role was also why these 3D WG foams exhibited lower surface-specific surface area (Supplementary Figure S11) and still resulted in a high adsorption capacity. The storage mechanism played a decisive role in the removal capacity. Therefore, two mechanisms could be hypothesized for the removal properties: adsorption and storage. For these 3D WG foams, the origins of the pollutant removal properties were not only from the adsorption capacity but also from the storage capacity. Hence, high-performance pollutant removal applications must fully utilize the combined effects of adsorption and storage.

Compared with the limitations of metal template chemical vapor deposition and ultrathin sheet assembling methods, the one-step heating of mixed commercial powders according to vesicant-assisted foaming is unrestricted by templates and rare starting materials, and hence had macroscopic yield. The intended introduction of foaming agents is very important for the foaming process. It not only guarantees the highly reproducible formation of foams but also results in hierarchical pores because of the matched decomposition thermodynamics between the BN precursors and the vesicants. The importance of such matched decomposition thermodynamics was also confirmed by the observed effects of the heating 

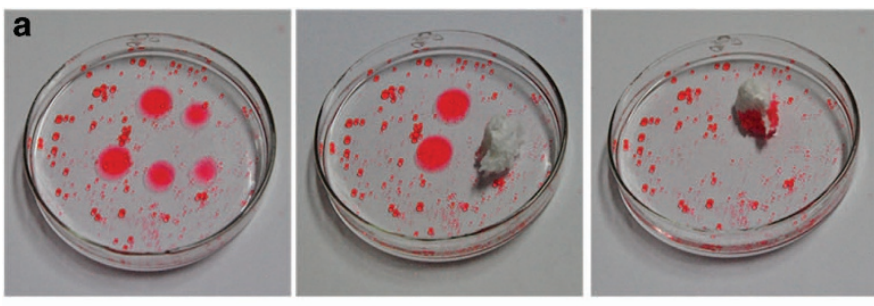

b

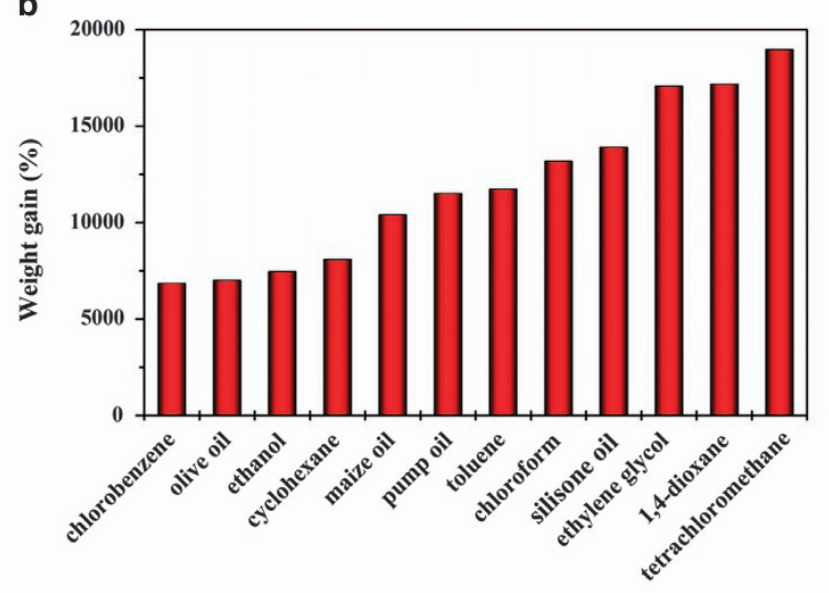

C

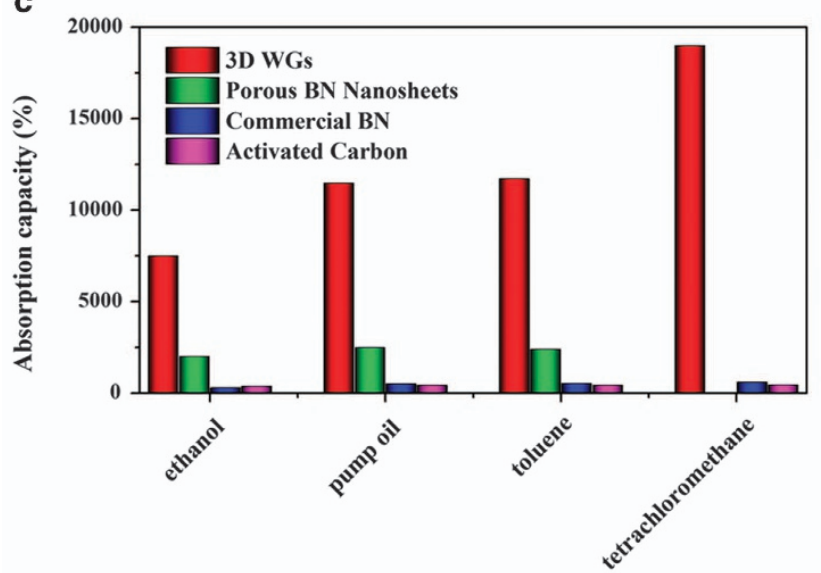

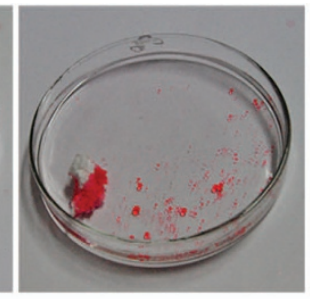

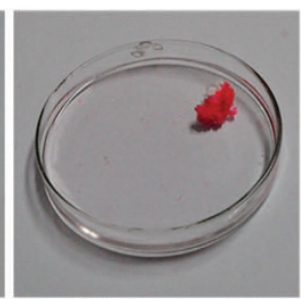

d

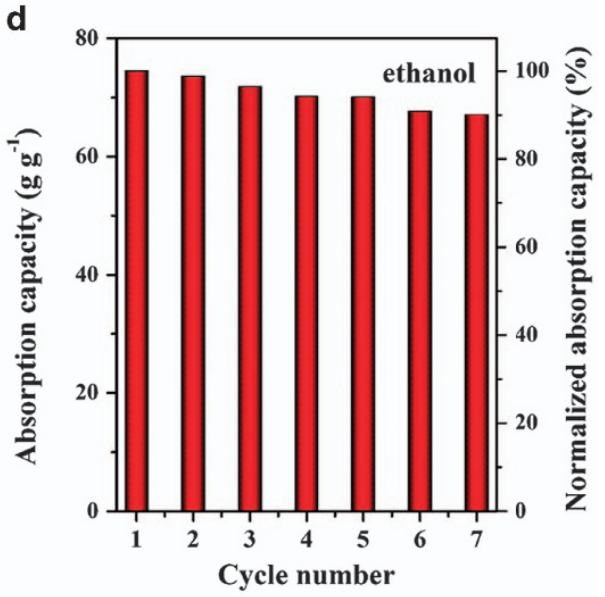

e

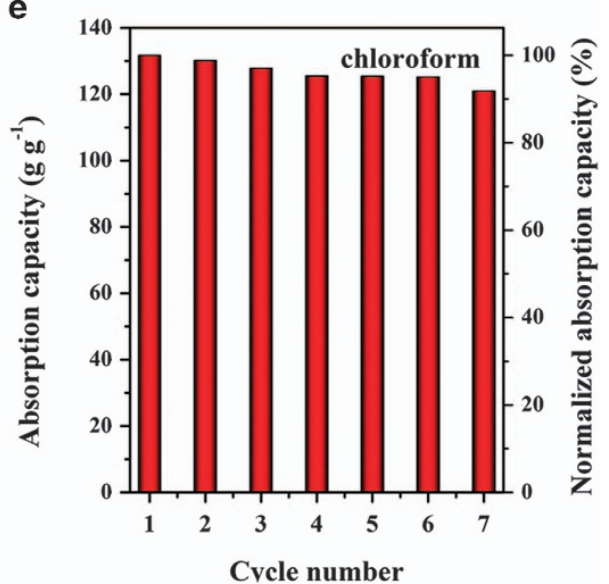

Figure 5 Outstanding adsorption performances of three-dimensional (3D) white graphene (WG) foams toward various organic contaminations. (a) Visual variation of the 3D WG-immersed chloroform solution within 1 min, showing the effective and rapid adsorption ability. (b) Adsorption capacities of the 3D WG foams toward various organic contaminations. (c) Comparison of the contamination adsorption capacities of the 3D WG foams with other typical sorbents, including commercial activated carbon. (d) Chloroform and (e) ethanol adsorption recyclability of 3D WG foams.

temperature and vesicant type on the final product, as shown in Supplementary Information (Supplementary Figures S12-S16).

\section{CONCLUSION}

We have developed a vesicant-assisted gas-foaming approach to synthesize vesicular structural 3D BN ceramic foams without any catalysts or templates. This technique could provide large-scale, highyield and ultralight 3D WG foams with hierarchical pores. This freesupporting BN foam consisted of a bubble wall of ultrathin BN sheets (single or several atomic layers) that were interconnected with each other by the framework. Moreover, the 3D WG foams presented extraordinarily high adsorption for organic solvents and oils. The adsorption capacity of the 3D WG foams was up to 70-190 times its own weight for organic pollutants and oils. The results revealed that the 3D WG foams have great potential as a high-performance pollutant scavenger for water treatment.

\section{CONFLICT OF INTEREST}

The authors declare no conflict of interest.

\section{ACKNOWLEDGEMENTS}

This work was financially supported in part by the National Basic Research Program of China (2014CB931700/2014CB931702), the National Natural Science Foundation of China (61222403), the Natural Science Foundation for Youths of Jiangsu Province of China (BK20140787), the China Postdoctoral Science Foundation funded project (2014M560425) and the Priority Academic Program Development of Jiangsu Higher Education Institutions (PAPD). 
1 Novoselov, K. S., Fal'ko, V. I., Colombo, L., Gellert, P. R., Schwab, M. G. \& Kim, K. A roadmap for graphene. Nature 490, 192-200 (2012).

2 Song, X., Hu, J. \& Zeng, H. Two-dimensional semiconductors: recent progress and future perspectives. J. Mater. Chem. C 1, 2952-2969 (2013).

3 Zeng, H., Zhi, C., Zhang, Z., Wei, X., Wang, X., Guo, W., Bando, Y. \& Golberg, D. "White Graphenes": boron nitride nanoribbons via boron nitride nanotube unwrapping. Nano Lett. 10, 5049-5055 (2010)

4 Song, L.., Liu, Z., Reddy, A. L., Narayanan, N. T., Taha-Tijerina, J., Peng, J., Gao, G., Lou, J., Vajtai, R. \& Ajayan, P. M. Binary and ternary atomic layers built from carbon, boron, and nitrogen. Adv. Mater. 24, 4878-4895 (2012)

5 Cao, X., Yin, Z. \& Zhang, H. Three-dimensional graphene materials: preparation, structures and application in supercapacitors. Energy Environ. Sci. 7, 1850-1865 (2014).

6 Li, C. \& Shi, G. Three-dimensional graphene architectures. Nanoscale 4, 5549-5563 (2012)

7 Lu, A., Hao, G. \& Sun, Q. Design of three-dimensional porous carbon materials: from static to dynamic skeletons. Angew. Chem. Int. Ed. 52, 7930-7932 (2013).

8 Bi, H., Xie, X., Yin, K., Zhou, Y., Wan, S., He, L., Xu, F., Banhart, F., Sun, L., Ruoff, R.S. Spongy graphene as a highly efficient and recyclable sorbent for oils and organic solvents. Adv. Funct. Mater. 22, 4421-4425 (2012).

9 Sun, H., Xu, Z. \& Gao, C. Multifunctional, ultra-flyweight, synergistically assembled carbon aerogels. Adv. Mater. 25, 2554-2560 (2013).

10 Chen, Z., Ren, W., Gao, L., Liu, B., Pei, S. \& Cheng, H. M. Three-dimensional flexible and conductive interconnected graphene networks grown by chemical vapour deposition. Nat. Mater. 10, 424-428 (2011).

11 Yin, J., Li, X., Zhou, J. \& Guo, W. Ultralight three-dimensional boron nitride foam with ultralow permittivity and superelasticity. Nano Lett. 13, 3232-3236 (2013).

12 Loeblein, M., Tay, R. Y., Tsang, S. H., Ng, W. B. \& Teo, E. H. T. Configurable threedimensional boron nitride-carbon architecture and its tunable electronic behavior with stable thermal performances. Small 10, 2992-2999 (2014).

13 Schlienger, S., Alauzun, J., Michaux, F., Vidal, L., Parmentier, J., Gervais, C. Babonneau, F., Bernard, S., Miele, P. \& Parra, J. B. Micro-, mesoporous boron nitride-based materials templated from zeolites. Chem. Mater. 24, 88-96 (2011).

14 Wu, T., Chen, M., Zhang, L., Xu, X., Liu, Y., Yan, J., Wang, W. \& Gao, J. Three-dimensional graphene-based aerogels prepared by a self-assembly process and its excellent catalytic and absorbing performance. J. Mater. Chem. A 1, 7612-7621 (2013).

$15 \mathrm{Hu}, \mathrm{H}$., Zhao, Z., Wan, W., Gogotsi, Y. \& J., Qiu J. Ultralight and highly compressible graphene aerogels. Adv. Mater. 25, 2219-2223 (2013)

16 Liu, Y., Ma, J., Wu, T., Wang, X., Huang, G., Liu, Y., Qiu, H., Li, Y., Wang, W. \& Gao, J. Cost-effective reduced graphene oxide-coated polyurethane sponge as a highly efficient and reusable oil-absorbent. ACS Appl. Mater. Interfaces 5, 10018-10026 (2013)

17 Rousseas, M., Goldstein, A. P., Mickelson, W., Worsley, M. A., Woo, L. \& Zettl, A. Synthesis of highly crystalline sp2-bonded boron nitride aerogels. ACS Nano 7, 8540-8546 (2013).

18 Höfer, R., Jost, F., Schwuger, M. J., Scharf, R., Geke, J., Kresse, J., Lingmann, H., Veitenhansl, R. \& Erwied, W. Ullmann's Encyclopedia of Industrial Chemistry (WileyVCH Verlag GmbH \& Co. KGaA, Düsseldorf, 2000).

19 Myers, D. Surfaces, Interfaces, and Colloids 295-316 (John Wiley \& Sons, Inc., New York, USA, 2002).

20 Banhart, J. Manufacture, characterisation and application of cellular metals and metal foams. Prog. Mater. Sci. 46, 559-632 (2001).

21 Banhart, J. Manufacturing routes for metallic foams. JOM 52, 22-27 (2000).

22 Studart, A. R., Gonzenbach, U. T., Tervoort, E. \& Gauckler, L. J. Processing routes to macroporous ceramics: a review. J. Ame. Ceram. Soc. 89, 1771-1789 (2006)
23 Withers, J. C., Kowbel, W. \& Loutfy, R. O. High Temperature Ceramic Matrix Composites 816-819 (Wiley-VCH Verlag GmbH \& Co. KGaA, Weinheim, FRG, 2006).

24 Wang, X., Zhi, C., Li, L., Zeng, H., Li, C., Mitome, M., Golberg, D. \& Bando, Y. "Chemical blowing" of thin-walled bubbles: high-throughput fabrication of large-area, few-layered BN and Cx-BN nanosheets. Adv. Mater. 23, 4072-4076 (2011).

25 Wang, X., Pakdel, A., Zhang, J., Weng, Q., Zhai, T., Zhi, C., Golberg, D. \& Bando, Y. Large-surface-area BN nanosheets and their utilization in polymeric composites with improved thermal and dielectric properties. Nanoscale Res. Lett. 7, 662 (2012).

26 Wang, X., del, A., Zhi, C., Watanabe, K., Sekiguchi, T., Golberg, D. \& Bando, Y. Highyield boron nitride nanosheets from 'chemical blowing': towards practical applications in polymer composites. J. Phys. Condens. Matter 24, 314205 (2012).

27 Frueh, S., Kellett, R., Mallery, C., Molter, T., Willis, W. S., King'ondu, C. \& Suib, S. L. Pyrolytic decomposition of ammonia borane to boron nitride. Inorg. Chem. 50, 783-792 (2010).

28 Gabbrielli, R., Meagher, A. J., Weaire, D., Brakke, K. A. \& Hutzler, S. An experimental realization of the Weaire-Phelan structure in monodisperse liquid foam. Phil. Mag. Lett 92, 1-6 (2012).

29 Ball, P. Scientists make the 'perfect' foam. Nat. News. (e-pub ahead of print 28 November 2011; doi:10.1038/nature.2011.9504).

30 Heim, K., Kumar, G. S. V., Garcia-Moreno, F., Manke, I. \& Banhart, J. Drainage of particle-stabilised aluminium composites through single films and Plateau borders. Colloid. Surface. A 438, 85-92 (2013).

31 Meeks, W. H. III \& Perez, J. The classical theory of minimal surfaces. B. Am. Math. Soc 48, 325-407 (2011).

32 Weng, Q., Wang, X., Zhi, C., Bando, Y. \& Golberg, D. Boron nitride porous microbelts for hydrogen storage. ACS Nano 7, 1558-1565 (2013).

33 Lian, G., Zhang, X., Zhang, S., Liu, D., Cui, D. \& Wang, Q. Controlled fabrication of ultrathin-shell BN hollow spheres with excellent performance in hydrogen storage and wastewater treatment. Energy Environ. Sci. 5, 7072-7080 (2012).

34 Lian, G., Zhang, X., Si, H, Wang, J., Cui, D. \& Wang, Q. Boron nitride ultrathin fibrous nanonets: one-step synthesis and applications for ultrafast adsorption for water treatment and selective filtration of nanoparticles. ACS Appl. Mater. Interfaces 5, 12773-12778 (2013).

35 Zhang, X., Lian, G., Zhang, S., Cui, D. \& Wang, Q. Boron nitride nanocarpets: controllable synthesis and their adsorption performance to organic pollutants. CrystEngComm 14, 4670-4676 (2012).

36 Lei, W., Portehault, D., Liu, D., Qin, S. \& Chen, Y. Porous boron nitride nanosheets for effective water cleaning. Nat. Commun. 4, 1777 (2013).

37 Wang, L., Ni, S.-Q., Guo, C. \& Qian, Y. One pot synthesis of ultrathin boron nitride nanosheet-supported nanoscale zerovalent iron for rapid debromination of polybrominated diphenyl ethers. J. Mater. Chem. A 1, 6379-6387 (2013).

38 Liu, D., Lei, W., Qin, S. \& Chen, Y. Template-free synthesis of functional 3D BN architecture for removal of dyes from water. Sci. Rep. 4 (2014).

(c) (i) This work is licensed under a Creative Commons Attribution 4.0 International License. The images or other third party material in this article are included in the article's Creative Commons license, unless indicated otherwise in the credit line; if the material is not included under the Creative Commons license, users will need to obtain permission from the license holder to reproduce the material. To view a copy of this license, visit http:// creativecommons.org/licenses/by/4.0/

Supplementary Information accompanies the paper on the NPG Asia Materials website (http://www.nature.com/am) 